TENDENCIAS

Revista de la Facultad de Ciencias

Económicas y Administrativas.

Universidad de Nariño ISSN-E 2539-0554

Vol. XXI No. 2 - 2do Semestre 2020,

Julio-Diciembre - Páginas 146-166

\title{
CARACTERIZACION Y MOTIVACIONES PARA EL EMPRENDIMIENTO FEMENINO EN MIPYMES DE VILLAVICENCIO - COLOMBIA
}

\section{CHARACTERIZATION AND MOTIVATIONS FOR FEMALE ENTREPRENEURSHIP IN MSMES IN VILLAVICENCIO - COLOMBIA}

\section{CARACTERIZAÇÃO E MOTIVAÇÕES PARA O EMPREENDEDORISMO FEMININO EM MIPYMES DE VILLAVICENCIO - COLÔMBIA}

\author{
RUIZ SANCHEZ_ María del Carmen; PEÑA GUERRERO_Jennifer Vanessa; \\ PRIETO CUBILLOS_Bryan Leonardo
}

Magíster en Administración, Instituto Tecnológico de Monterrey. Docente investigadora, Universidad de los Llanos. E-mail: mariacruiz@unillanos.edu.co, Colombia.

Profesional en Contaduría Pública, Facultad de Ciencias Económicas, Universidad de los Llanos, Email: jennifer.pena@unillanos.edu.co, Colombia.

Profesional en Contaduría Pública, Facultad de Ciencias Económicas, Universidad de los Llanos, Email: bryan.prieto@unillanos.edu.co, Colombia.

Recibido: 30 de enero de 2020

Aprobado: 6 de julio de 2020

DOI: https://doi.org/10.22267/rtend.202102.145

\section{RESUMEN}

Este artículo tiene como objetivo explorar acerca de las características y motivaciones que generan las iniciativas de emprendimiento de las mujeres de la comuna cinco de la ciudad de Villavicencio. Estableciendo como condición para develar dichas características y motivos, la formalización de las unidades empresariales. La metodología empleada responde a un enfoque mixto; mediante el tipo cuantitativo se efectuó la caracterización de las 16 unidades empresariales que se encontraron 
lideradas por mujeres en el contexto de estudio y a través del método cualitativo se profundizó en las motivaciones del emprendimiento directamente de las seis mujeres que aceptaron responder una entrevista semiestructurada. Los resultados evidencian que las emprendedoras, en su mayoría iniciaron por necesidad y con fines de auto empleo; no obstante, por la experiencia y el conocimiento adquirido la gran mayoría ha logrado la sostenibilidad de sus negocios, al superar una existencia de más de diez años, posibilitando su categorización como empresarias establecidas.

Palabras clave: emprendimiento femenino; caracterización; motivación; MIPYMES; entorno.

JEL: J16; M10; M13; M14; M20

\section{ABSTRACT}

This article aims to explore the characteristics and motivations that entrepreneurship initiatives generate for women in commune five in the city of Villavicencio. Establishing as a condition to reveal these characteristics and motives, the formalization of business units. The methodology used responds to a mixed approach; By means of the quantitative type, the characterization of the 16 business units that were found to be led by women in the study context, was carried out and through the qualitative method, the motivations of entrepreneurship were deepened directly from the six women who agreed to answer a semi-structured interview. The results show that the entrepreneurs, for the most part, started out of necessity and for self-employment purposes; However due to the experience and knowledge acquired, the vast majority have achieved the sustainability of their businesses, overcoming an existence of more than ten years, making it possible to classify them as established entrepreneurs.

Keywords: female entrepreneurship; characterization; motivation; MSMEs; environment.

JEL: J16; M10; M13; M14; M20

\section{RESUMO}

Este artigo tem como objetivo explorar as características e motivações que as iniciativas de empreendedorismo geram para as mulheres na comuna cinco na cidade de Villavicencio. Estabelecendo como condição para revelar essas características e motivos, a formalização das unidades de negócios. A metodologia utilizada responde a uma abordagem mista; Por meio do tipo 
quantitativo, foi realizada a caracterização das 16 unidades de negócios encontradas lideradas por mulheres no contexto do estudo e, pelo método qualitativo, foram aprofundadas as motivações do empreendedorismo diretamente das seis mulheres que concordaram em responder a uma entrevista semiestruturada. Os resultados mostram que os empreendedores, na maioria das vezes, começaram por necessidade e por conta própria; No entanto, devido à experiência e ao conhecimento adquiridos, a grande maioria alcançou a sustentabilidade de seus negócios, superando a existência de mais de dez anos, possibilitando classificá-los como empreendedores estabelecidos.

Palavras-chave: empreendedorismo feminino; caracterização; motivação; MPME; meio ambiente. JEL: J16; M10; M13; M14; M20

\section{INTRODUCCIÓN}

El emprendimiento es considerado un agente generador de impacto en el desarrollo socio-económico de las naciones, especialmente en los países emergentes, en los cuales aparece como una alternativa para la generación de ingresos dada la escasez de oportunidades para encontrar un empleo debidamente formalizado, incorporándose además en los planes y programas de política pública como herramienta eficaz para disminuir la tasa de desempleo e inyectar dinamismo a la economía ayudando al crecimiento y sostenibilidad del tejido empresarial a través de la interrelación con otras organizaciones empresariales.

La revisión de la literatura permitió establecer diversas definiciones y tipos de emprendimiento, entre los que sobresalen el emprendimiento de oportunidad y el emprendimiento por necesidad; en este sentido, se encontraron como principales motivaciones para que las mujeres se adentren en prácticas emprendedoras, como lo plantean León, Aguas y Rojas, (2018) "la necesidad de independencia económica y el obtener ingresos por sus propias fuentes para aportar a sus familias y sentirse productivas para la sociedad, por esta razón ven en el emprendimiento la oportunidad de cubrir sus necesidades y poder solventar sus problemas" (p.4).

Desde los planteamientos del Global Entrepreneurship Monitor (GEM), que muestra la Tasa de Actividad Emprendedora (TEA, por su sigla en inglés), Colombia se ha mantenido en una cultura 
emprendedora relativamente estable durante los últimos cinco años, con un indicador "TEA de 18,9\%, compuesta en mayor proporción por emprendedores nacientes $(60 \%)$ en relación con nuevos empresarios (40\%)" (GEM, 2017, p. 5); igualmente, las mediciones de oportunidad y necesidad para el mismo año, reflejan que en el país se da en mayor grado el emprendimiento de oportunidad (14,5\%) que el de necesidad (3,5\%), en tanto que para América Latina y el Caribe los índices son del 13,1\% y $5,2 \%$, respectivamente (GEM, 2017, p.25).

Cabe destacar que, en el estudio del emprendimiento, el género emerge como una de las categorías relevantes para su contextualización y análisis. Es importante destacar el rol del emprendimiento femenino en el desarrollo económico de una región, según datos del Banco Mundial (2010, citado en Elizundia, 2015) "Alrededor del 35-40\% de los micro, pequeños y medianos negocios son operados por Mujeres" (p. 472).

Los indicadores de emprendimiento en Colombia, según datos del GEM (2017), evidencian una reducción importante en las diferencias del emprendimiento de género durante el periodo 2013-2017, al pasar de 13,2\% al 1\%, presentándose para el 2017 en un nivel del 19,2\% para los hombres y del $18,2 \%$ para las mujeres.

Del contexto de estudio, Fierro (2007, citado en Obando-Bastidas, Herrera-Sarmiento y RodríguezLadino, 2016), resalta a Villavicencio como una ciudad con expectativas de crecimiento en pro de la consolidación de un sector empresarial fuerte y diversificado; también Arosa, Giraldo, Pinilla, Rodríguez, y Vargas (2010, citado en Obando et al., 2016), exponen acerca de la existencia aproximada de 9.748 empresas formalizadas, de ellas el 97.34\% son MIPYMES, y según la Ley 905 del 2004, que fomenta la promoción, formación y funcionamiento de estas unidades empresariales, son las que en mayor proporción generan empleo y contribuyen al desarrollo de un país.

Al distinguir a las mujeres como sujeto activo de emprendimiento en la comuna cinco de la ciudad de Villavicencio, en este artículo se exploran las características y las motivaciones que impulsaron a la creación de empresa en articulación con la contribución efectuada a la sociedad, en la búsqueda de reconocer la influencia de dichas motivaciones en las iniciativas de emprendimiento y el surgimiento de nuevas empresarias. 
El artículo se desarrolla en cinco apartados: la presente introducción que muestra la importancia del emprendimiento y las posibles motivaciones para que se generen iniciativas de emprendimiento femenino; le sigue una aproximación teórica y conceptual, en ella se presenta la revisión de la literatura que se relaciona con el desarrollo del emprendimiento dando prelación al enfoque de género; en el siguiente apartado se presenta la metodología que explica la utilización de un enfoque mixto para llevar a cabo el proyecto, con énfasis en la aplicación de una encuesta semi-estructurada para la obtención de los resultados; posteriormente se exponen y discuten los resultados, a partir de la presentación de cinco categorías y finalmente se presentan las conclusiones.

\section{REFERENTES TEÓRICOS}

El término emprendedor, visto desde la definición de la Real Academia Española (2018), se considera un adjetivo, refiriéndose a: "Que emprende con resolución acciones o empresas innovadoras”. Su devenir histórico se remonta al año 1755, según los planteamientos de Pereira (2003, citado por Bucardo, Saavedra y Camarena, 2015), al ser propuesto originalmente el termino por Richard Cantillón como "entrepreneur" para referirse a una persona "caracterizada por comprar productos a precios conocidos para venderlos en el mercado a precios desconocidos” (p. 99), enfatizando además que es el emprendedor quien soporta los riesgos de su actividad.

No obstante, la literatura le asigna a Shumpeter un papel preponderante en el desarrollo de la temática del emprendimiento, por contarse entre los primeros que visualiza su importancia en la ciencia económica, además de vincularlo con la transformación de ideas y la innovación, su legado se cimenta en el principio de la destrucción creativa que se genera por las actividades de innovación, cimentadas en características de creatividad y liderazgo, realizadas por los emprendedores (Bucardo et al., 2015; Castiblanco, 2013; Marulanda, Correa y Mejía, 2009; Velásquez, 2009).

La construcción conceptual y normativa se ha alimentado a lo largo del tiempo por múltiples autores, dando origen a diferentes tendencias y modelos de emprendimiento, que han acentuado su importancia hasta considerarlo un elemento esencial en el proceso de creación de empresa y una herramienta eficaz

para crear empleo, que posibilita la generación de ingresos para los ciudadanos y mejora la competitividad y el bienestar de la sociedad; pues los emprendedores son llamados a identificar, 
valorar y ejecutar las oportunidades de negocio, haciéndolo participe del desarrollo económico y social de cualquier país. Así lo expone Valencia (2012), quien además relaciona el emprendimiento con el autoempleo considerando que este se impulsa a partir de políticas públicas en atención a que "El autoempleo se genera por los empresarios o tomadores de riesgo: aquellas personas que por su afán de ganancia o por tener una idea que creen puede revolucionar el mundo son los llamados a autoemplearse" (p. 107).

Además de relacionar el emprendimiento con la innovación y la renovación de ideas y procesos (Valencia, 2012; Burgos, Pombosa y López 2017), para efectos del presente artículo merecen resaltarse dos concepciones de emprendimiento: la de Veciana (1999), quien le asigna un enfoque socio-antropológico al plantear que las motivaciones para emprender están condicionadas por el comportamiento humano y el medio ambiente en el que se desenvuelve y, la definición emanada por el GEM: "cualquier intento de nuevo negocio o la creación de empresas, tales como trabajo por cuenta propia, una nueva organización empresarial o la expansión de un negocio existente, bien sea de un individuo, de un equipo de personas" (GEM, 2017, p.9), a partir de la cual se realizan múltiples análisis de contextualización, caracterización y medición en diferentes países.

En este sentido, el GEM (2017) para relacionar el emprendimiento con la sostenibilidad de la empresa lo sectoriza en cuatro fases:

- Emprendedor potencial, quien tiene alguna intención de emprender.

- Emprendedor naciente, apenas iniciado el negocio con un periodo no superior a tres meses.

- Nuevo empresario, quien ha logrado mantener el negocio en un lapso de tres a 42 meses.

- Empresario establecido, aquel que ha logrado estabilizar el negocio y contratar empleados por un tiempo superior a 42 meses.

Aunque tradicionalmente la actividad emprendedora ha sido asociada con la masculinidad, es precisamente el GEM quien propicia la labor de efectuar la diferenciación del emprendimiento de género, incluyendo el emprendimiento liderado por mujeres y, según la Organización para la Cooperación y el Desarrollo Económicos (OCDE, por su abreviatura en inglés), este ha ido en crecimiento hasta convertirse en una fuente importante de innovación y generación de nuevos empleos (sf, citado en Avolio y Di Laura, 2017). 
El emprendimiento femenino se presenta como resultado de diversas motivaciones y situaciones originadas en el entorno del emprendedor tales como: las situaciones económicas, familiares, sociales o vivencias que generan en la mujer necesidades e iniciativas para emprender (Sharpero,1985; Ajzen, 1990, citado por Marulanda, Montoña y Vélez, 2014), en tanto que otros autores como Stoner y Fry (1982 citado en Navarro, 2016), han optado por hacer una clasificación para “emprender por necesidad (push) o por oportunidad (pull)" (p. 395) aduciendo que ello puede incidir en la perdurabilidad y el éxito de una empresa.

De lo anterior, puede inferirse que las motivaciones tienen relación directa con las condiciones específicas del entorno en el que se realizan las actividades de emprendimiento, como lo plantea Gartner (1985, citado por Pereira, 2003), quien señala al entorno como uno de los cuatro elementos presentes en el espíritu emprendedor; igualmente, Azqueta (2019), lo señala como agente dinamizador del entorno, por lo cual se considera elemento de gran importancia para el caso del emprendimiento femenino. Igualmente, el conocimiento del entorno, ha sido señalado como uno de los trece aspectos que garantizan la perdurabilidad de las empresas, (Castro, Farfán y Olis, 2013), entendida ésta última como la capacidad de una empresa para sostenerse en el mercado.

\section{METODOLOGÍA}

El método responde a un enfoque descriptivo de tipo mixto, cuantitativo y cualitativo, desde los planteamientos de Pita y Pétergas (2002) “La investigación cuantitativa es aquella en la que se recogen y analizan datos cuantitativos sobre variables" (p.1). La investigación cualitativa se encarga de recolectar información mediante "registros narrativos de los fenómenos que son estudiados mediante técnicas como la observación participante y las entrevistas no estructuradas" (Pita y Pétergas, 2002, p.1).

Desde el enfoque cualitativo Boeije (2010, citado en Ugalde y Balbastre, 2013) afirma que "los científicos en ciencias sociales aprueban el uso de metodologías cualitativas en estudios constructivistas porque dan participación al ser humano (objeto de disertación), en lugar de tratarlo como un sujeto pasivo, como se hace en las investigaciones cuantitativas" (p. 182). 
El proceso de recolección de información se realizó en tres fases:

La primera fase consistió en una visita de campo a la comuna con el fin de identificar las micro, pequeñas y medianas unidades empresariales que cumplían dos requisitos: lideradas por mujeres y estar debidamente formalizadas mediante inscripción en la Cámara de Comercio de la ciudad y exhibición del registro único tributario (RUT). Esta fase permitió detectar 32 establecimientos formalizados, de los cuales 16 (50\%), cumplían con la condición de ser liderados por mujeres.

La segunda y tercera fases se desarrollaron para la obtención de información primaria. La segunda fase permitió efectuar la caracterización a las 16 unidades empresariales lideradas por mujeres (50\%), que se constituyeron en la unidad de análisis.

La tercera y última fase se desarrolló con la finalidad de profundizar en las motivaciones para el emprendimiento, para ello se solicitó a las integrantes de la unidad de análisis la autorización para profundizar la información con el objetivo de establecer las motivaciones que originaron el emprendimiento, que fue aceptada únicamente por seis emprendedoras que conformaron la unidad de trabajo.

En coherencia, con los objetivos propuestos y las fases desarrolladas se determinó la aplicación de dos instrumentos: encuesta y entrevista semi-estructurada. La encuesta se conformó por 28 preguntas agrupadas en cuatro apartados: el primero se encarga de recolectar la información que identifica la unidad empresarial y su ubicación; el segundo se ocupa de obtener la información de la empresaria, el tercero muestra los datos del núcleo familiar y finalmente el ultimo indaga acerca de las características de la unidad empresarial, las razones y el tipo de emprendimiento. El análisis de la información proporcionada por la encuesta se sistematizó a partir la base de datos SPSS versión 19 con el objeto de cuantificar los resultados por medio de tablas y figuras.

La entrevista semi-estructurada se realizó con el objetivo de profundizar en las motivaciones y experiencias directamente de las emprendedoras, a partir de 42 interrogantes que pretendieron extractar el mayor grado de información; una vez terminada esta fase se procedió a efectuar la transcripción de las entrevistas y a elaborar una lista de las motivaciones expresadas, con las cuales se construyeron las principales categorías. 
La codificación de las respuestas permitió la agrupación en cuatro categorías, tres de ellas sobresalen directamente: iniciativa propia, necesidad de ingresos y necesidad de independencia y una cuarta que se denominó varias, en la cual se recogieron otra serie de respuestas que surgieron con menor frecuencia.

La recolección de información se llevó a cabo, en la comuna cinco, por ser la más grande de las ocho que comprenden la Ciudad de Villavicencio, al conformarse por 108 de los 435 barrios de la ciudad, que se constituye en el territorio contexto de estudio, como se muestra en la Figura 1.

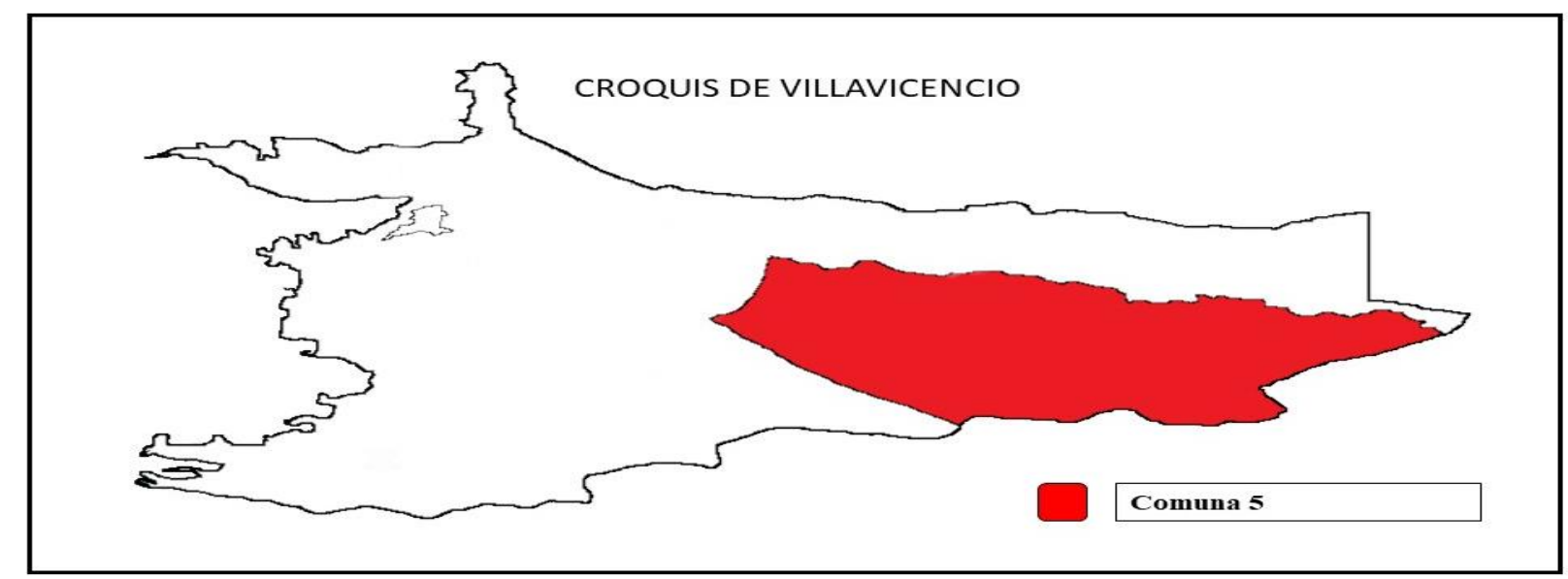

Figura 1. Comuna cinco de la ciudad de Villavicencio.

Fuente: Secretaría de Planeación Departamental (2017).

\section{DISCUSIÓN}

Se exponen los resultados en dos fases: en la primera se hace una caracterización de las unidades empresariales que conformaron la unidad de análisis y en la segunda se exponen las motivaciones para el emprendimiento. Cabe advertir que dichas motivaciones surgen del cruce de respuestas de los dos instrumentos aplicados a las empresarias, sin embargo, la entrevista sirvió para profundizar en las motivaciones que generaron las iniciativas de emprendimiento y conformar las subcategorías. 


\section{Caracterización}

De las características sociodemográficas en que se desarrollaron las iniciativas de emprendimiento de la unidad de análisis (16 empresarias), sobresalen: la edad de las empresarias, dado que la gran mayoría (62\%) se encuentra entre los 36 y 55 años (Ver Figura 2), y la condición de mujer cabeza de hogar que manifiestan las dos terceras partes de las mujeres participantes, que en su mayoría son mujeres separadas a cargo del núcleo familiar (75\%).

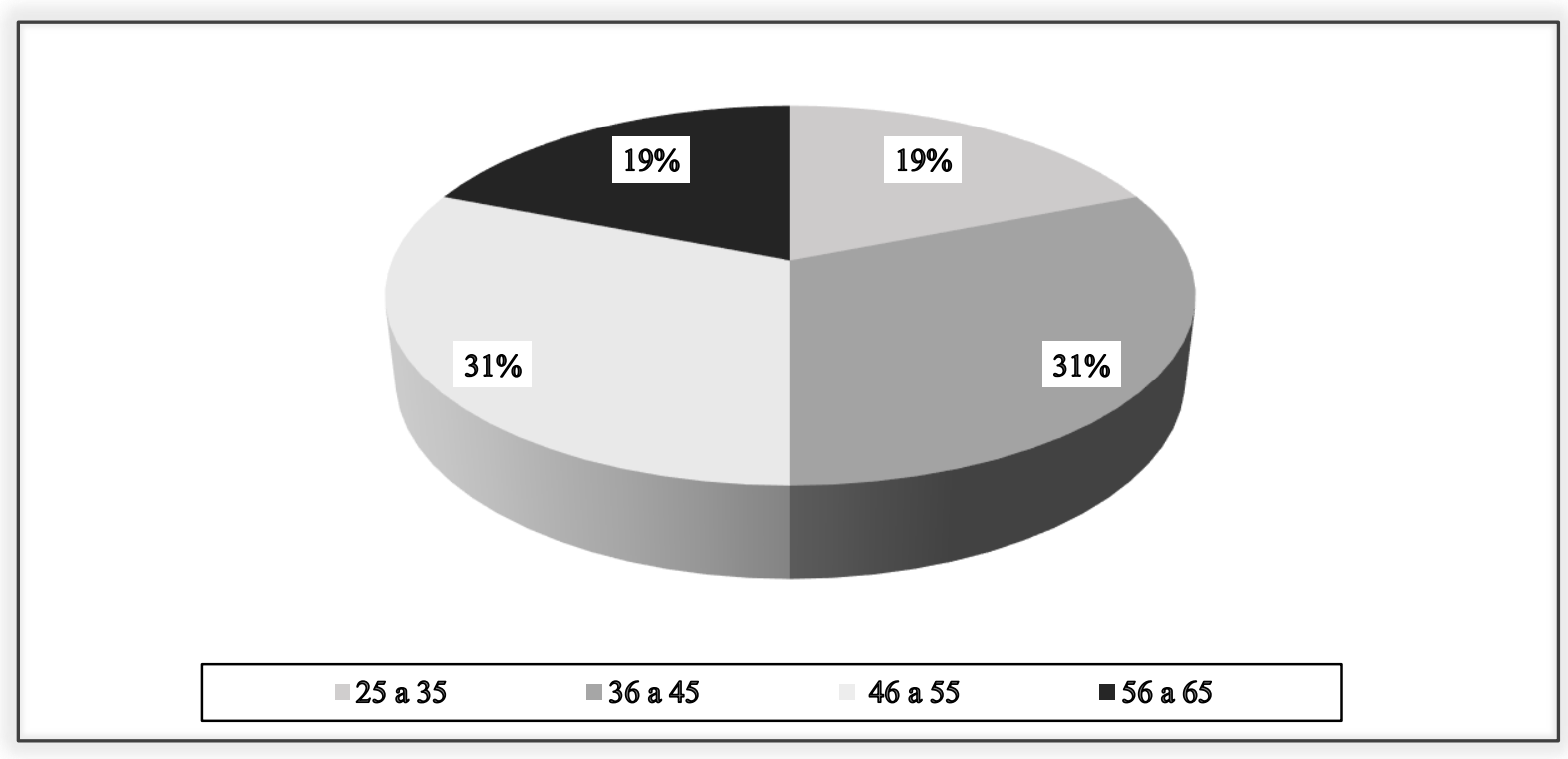

Figura 2. Edad de las empresarias.

Fuente: elaboración propia.

Igualmente, el perfil educativo, al denotarse que más de la mitad de las empresarias ha realizado estudios de nivel superior (57\%), en mayor grado a nivel técnico y tecnológico, en tanto que el $6 \%$ presentan nivel profesional y el 6\% ostenta título de posgrado; evidenciándose también que una tercera parte de la unidad de análisis apenas terminó sus estudios de nivel secundario.

No obstante, la característica de mayor relevancia la constituye la experiencia de las empresarias, en atención a que la mayor parte de la unidad de análisis (89\%) tiene más de cinco años desempeñándose en su labor; es importante señalar que sobresale el rango de diez a veinte años de experiencia con una 
participación del 63\%, lo que indica que no son empresarias nacientes, por el contrario, son empresarias establecidas, que han logrado el sostenimiento de sus negocios en el mercado.

Otro aspecto a resaltar es el sector económico en el que se desempeñan, el estudio evidenció que el sector comercial agrupa a la mayoría de las unidades empresariales analizadas (67\%); las actividades más practicadas son la comercialización de productos de belleza, almacenes para la venta de ropa, supermercados y tienda de regalos. Le siguen en orden de importancia las actividades de servicios (22\%), que presenta a las actividades de belleza y a los restaurantes con mayor ejercicio en el territorio de estudio; finalmente se encuentran actividades industriales (11\%), correspondiendo a las que tienen alguna relación con la alimentación, tales como como la producción de panadería y pastelería y productos para el consumo familiar como la fabricación de arepas.

También vale señalar el tamaño de las empresas, de acuerdo a las categorías establecidas en la Ley 905 de 2014, que establece como elementos para definir el tipo de organización tanto el nivel de activos como el número de empleados; con base en el parámetro de planta de personal, empresas con menos de diez empleados se clasifican como microempresas, por lo cual puede decirse que el $87 \%$ de las unidades empresariales corresponde a este tipo de organizaciones y aquellas que incorporan más de 10 y menos de 50 empleados se designan como pequeñas empresas (13\%), como se muestra en la Figura 3.

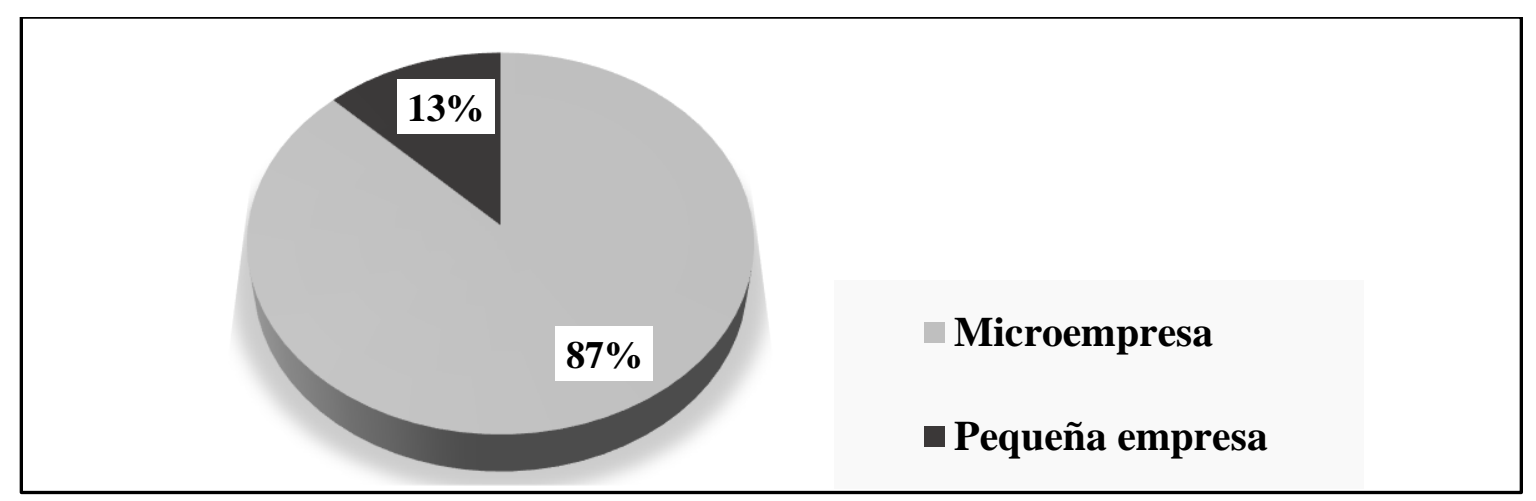

Figura 3. Tamaño de las empresas.

Fuente: elaboración propia. 
Otro factor de importancia, que merece señalarse es el tiempo de existencia de las organizaciones, la gran mayoría (76\%) sobrepasa los cinco años de existencia, aspecto que denota el sostenimiento de los negocios en el mercado; elemento que permite catalogarlos como negocios establecidos, capaces de mantenerse en el mercado y de cumplir con el pago de sus obligaciones, incluyendo la planta de personal (Figura 4).

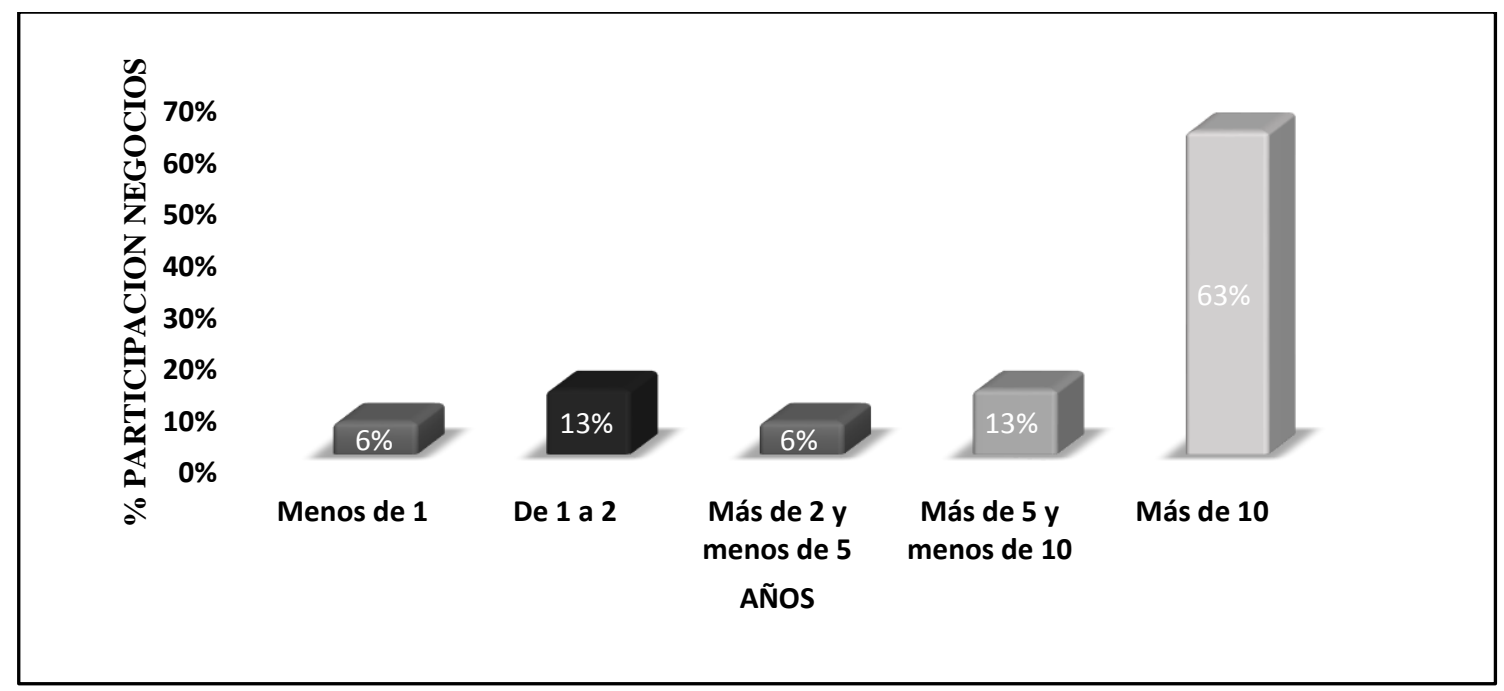

Figura 4. Tiempo de funcionamiento de la unidad empresarial.

Fuente: elaboración propia.

En la búsqueda de profundizar en la importancia de estas unidades de negocio para cada una de las familias y cómo la intencionalidad de obtener un empleo puede convertirse en una motivación para el emprendimiento en Villavicencio, se indagó acerca del número de personas que emplean las unidades empresariales y cuantas de ellas pertenecen al núcleo familiar; obteniéndose que en las dos terceras partes se generan de dos a trece empleos, de los cuales al menos la mitad son asumidos por las personas del núcleo familiar; en tanto, la otra tercera parte de los negocios estudiados solo posibilita la creación de un empleo, que es asumido en el 56\% de los casos por las propias emprendedoras (Figura 5). 


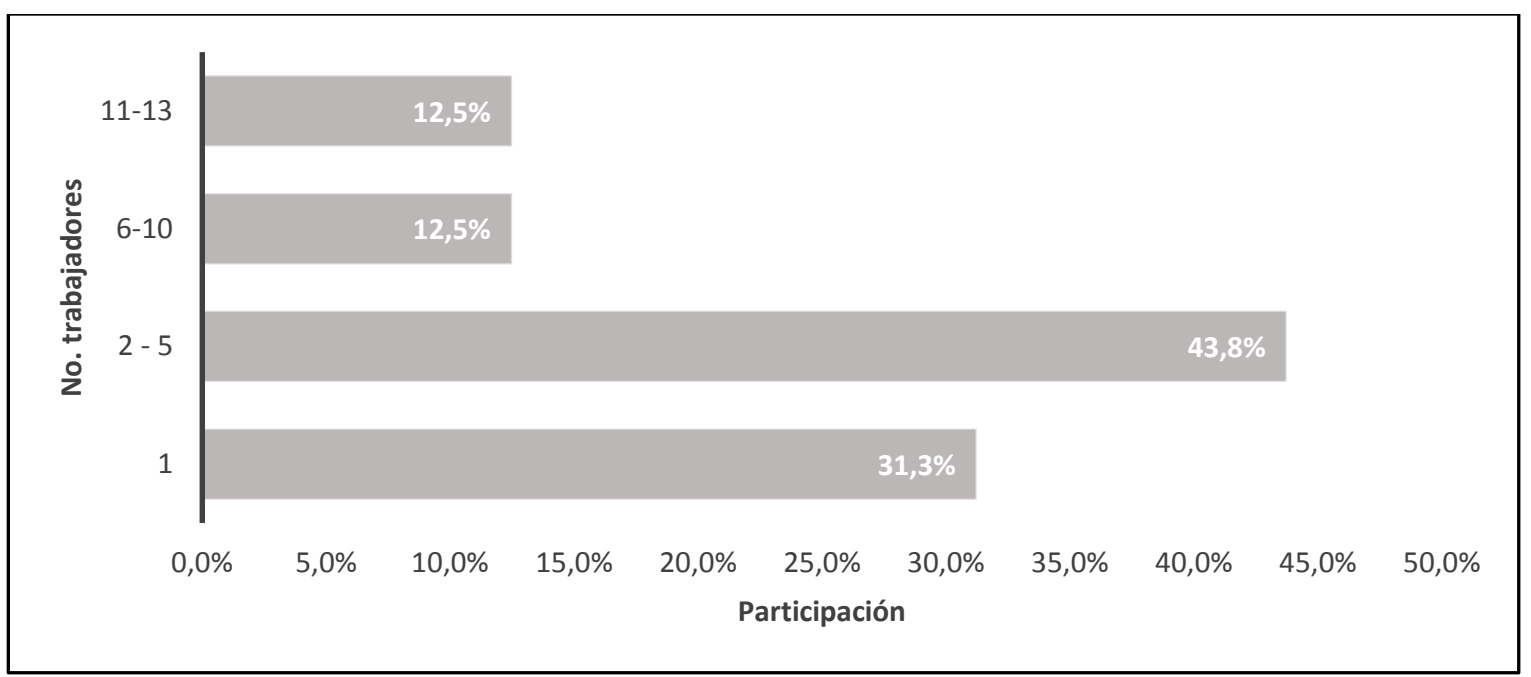

Figura 5. Planta de personal del negocio.

Fuente: elaboración propia.

\section{Motivaciones para crear empresa}

Como se indicó en la metodología, las principales motivaciones para las iniciativas de emprendimiento se agruparon en cuatro categorías que comprenden a todas las unidades empresariales analizadas: iniciativa propia, necesidad de ingresos, necesidad de independencia y otras justificaciones de menor participación que fueron agrupadas en una categoría que se denominó varias, como se puede observar en la Tabla 1.

\section{Tabla 1}

Categorización de las motivaciones para emprender

\begin{tabular}{lll}
\hline \multicolumn{1}{c}{ Categoría } & \multicolumn{1}{c}{ Subcategoría } \\
\hline Iniciativa propia & - Amor por la creación de empresa \\
& - Deseo de superación \\
& - Influencia de la familia \\
Necesidad de ingresos & - Sxperiencia en la actividad \\
& - Desemimiento de la familia \\
& - Perdida reiterada de empleos \\
\hline
\end{tabular}




\begin{tabular}{ll}
\hline Necesidad de independencia & $\bullet$ Equilibrar tiempo laboral y familiar \\
& $\bullet$ Aversión a la dependencia \\
& $\bullet$ Conocimiento del oficio \\
Otras & $\bullet$ Tradición familiar \\
& $\bullet$ Experiencia y conocimiento del oficio \\
& $\bullet$ Búsqueda de seguridad personal y familiar \\
\hline
\end{tabular}

Fuente: elaboración propia.

Las subcategorías permitieron establecer que las necesidades de la familia están presentes de una u otra forma en todas las motivaciones que generaron las iniciativas de emprendimiento de las mujeres en el contexto de estudio, como se evidencia en algunas de las respuestas.

Entrevistada 1: "Mi principal motivación es sostener a mi familia, del pueblo de donde venía había mucha violencia y cuando me vine para acá estaba estudiando y poco a poco fui consiguiendo lo que tengo".

Entrevistada 2: "Esto lo tengo es por mi hijo, que es administrador de empresas".

Entrevistada 3: "La familia lo es todo, Yo tenía apenas nada, mis hijos que apenas salían del colegio venían y me ayudaban".

La principal motivación para el emprendimiento femenino en las unidades analizadas lo ocupa la categoría relacionada con la iniciativa propia (30\%), que se sustenta en mayor grado con el amor por el emprendimiento, la creación de empresa y la realización personal.

En segundo lugar, emergió la necesidad de ingresos de las mujeres (22\%), directamente relacionada con las necesidades del núcleo familiar y la falta de empleo, que obliga a las mujeres a generar alternativas de autoempleo para la consecución de ingresos.

En tercer lugar, se ubicó la necesidad de independencia (19\%), que surge fundamentalmente de la posibilidad de manejar el tiempo para combinar las obligaciones familiares con la atención del 
negocio, el conocimiento y la experiencia en la actividad sumada al rechazo a la dependencia de un patrón o un empleo, lo que le imprime cierta sensación de seguridad a las mujeres.

En cuarto lugar, se agruparon una serie de diversas razones como el desarrollo de la actividad por tradición familiar, lo que les concede determinado grado de conocimiento y experiencia en el oficio, el conocimiento previo del oficio adquirido en anteriores empleos finalmente, situaciones familiares particulares y problemas de orden social (violencia y desplazamiento), cabe anotar que las empresarias que manifiestan haber vivido este tipo de problemáticas son las más renuentes a brindar información y no aceptaron formar parte de la unidad de trabajo para conceder la entrevista.

Es importante resaltar las expresiones efectuadas por las mujeres emprendedoras, pues la mayoría de ellas relata que el hecho de crear su propia empresa además de considerarse la principal fuente de ingresos y posibilitar mayor solvencia económica, presenta otras ventajas, entre las que sobresalen: la facilidad en el manejo de su tiempo para atender aspectos familiares y personales, posibilidades para estudio o capacitación y lograr mejores relaciones interpersonales.

Las cuatro categorías que plantean las motivaciones para el emprendimiento femenino en el contexto válido de estudio, pueden asociarse con variables ampliamente identificadas en la revisión de la literatura; en primer lugar se detecta que el emprendimiento en las MIPYMES analizadas se genera por necesidad, confirmando los planteamientos de León et al., (2018) y Shapero (1985, citado en Marulanda et al., 2014), quienes argumentan que una de las principales motivaciones para el emprendimiento es la necesidad de independencia económica y de generación de ingresos para solventar las necesidades familiares. Igualmente, se confirma lo propuesto por Frank (2012, citado en Castiblanco, 2013), quien a partir de su investigación determina que una de las motivaciones principales de la mujer al momento de emprender, es su familia (Figura 6). 


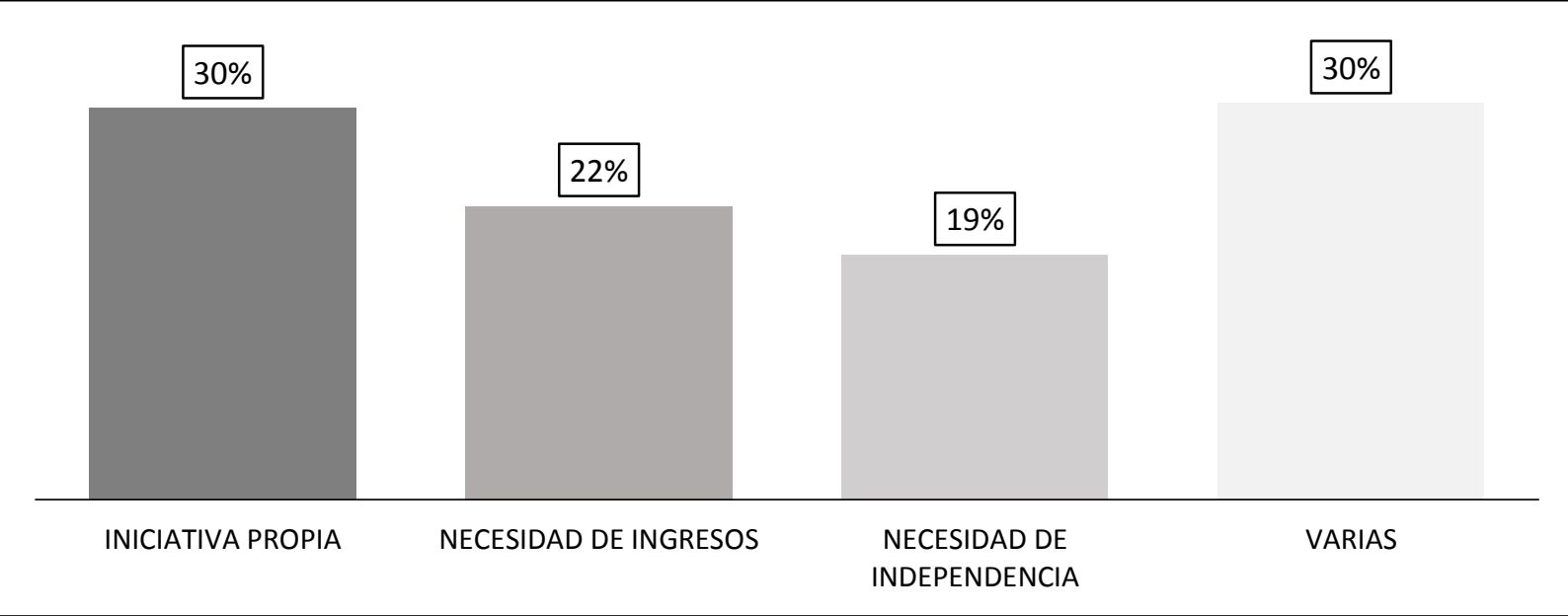

Figura 6. Motivaciones para crear empresa.

Fuente: elaboración propia.

Vale la pena anotar que las condiciones del país juegan un papel primordial en las motivaciones ya que factores externos como el desempleo y la seguridad influyen en la búsqueda de alternativas para la consecución de ingresos, que se suman a condiciones particulares de las mujeres como son la familia, algunas incursiones previas en la actividad y las ganas de superación se constituyen en elementos que incentivan el emprendimiento femenino, para generar el sustento para el hogar, permitiendo cubrir gran parte de sus gastos familiares, como lo son: la salud, estudio, vivienda, recreación, entre otros, fundamentales para su calidad de vida.

Igualmente, los resultados permiten inferir que el citado emprendimiento por necesidad conllevó al autoempleo, como lo plantearon algunas de las empresarias inicialmente su negocio solo logró proporcionar posibilidades de ingresos para la propietaria, situación en la que todavía se mantiene el 31,3\% de las MIPYMES analizadas. Lo que permite establecer que el emprendimiento, a través del autoempleo, se convierte en una solución a los problemas que presenta el país en un aspecto de importancia como lo es el empleo, aspecto que sustenta los planteamientos de la OCDE (2016) "Debido a problemas estructurales del mercado laboral en Colombia se evidencia una tasa elevada de autoempleo, el 52\% de la población que trabaja como independiente" (p. 14). Adicionalmente, los datos del estudio permiten resaltar que dos tercios de las empresas que se crearon con fines de autoempleo, y con el crecimiento del negocio fueron vinculando otras personas hasta considerarse en la actualidad como generadoras de empleo (Más de seis y hasta 13 empleados). 
Datos relevantes del estudio destacan que dos tercios de las emprendedoras han logrado mantenerse en el mercado por más de cinco años efectuando el pago de salarios a su planta de personal, lo que según el GEM (2017), permite ubicarlas en la categoría de empresarias establecidas, al superar ampliamente los 42 meses de despegue propuestos para ella. En este aspecto, cobra vital importancia la experiencia como factor que contribuye a la perdurabilidad, Dencker, Gruber y Shah (2009, citado en Simón, Revuelto y Medina, 2012), exponen que la experiencia y el conocimiento previo tiene una relación positiva con la supervivencia de las empresas, se debe destacar que a medida que se avanza en el camino los empresarios van adquiriendo conocimientos que le permiten desarrollar estrategias y tomar mejores decisiones para iniciar el negocio, fortalecerlo y lograr que este sea perdurable en el tiempo.

El estudio también permite afirmar que, en atención al tamaño de la planta de personal utilizada por las unidades, estas pueden catalogarse como MIPYMES según lo dispuesto en la Ley 905 de 2004, dado que el 100\% de las empresas de la comuna 5 son micro y pequeñas empresas. De acuerdo con la Asociación Colombiana de las Micro, Pequeñas y Medianas empresas (ACOPI, 2017), las Mipyme representan más del 95\% del tejido empresarial del país, este segmento agrupa aproximadamente entre el 65\% y el 67\% de los empleados y generan el 28\% del PIB, las MIPYMES estudiadas contribuyen con dichos indicadores ayudando a combatir la pobreza, el subempleo y el desempleo de la ciudad.

\section{CONCLUSIONES}

La principal motivación para el emprendimiento femenino en la comuna 5 de Villavicencio es la necesidad, confirmándose los planteamientos de Stoner y Fry (1982 citado en Navarro, 2016), al establecerse que la mayoría de los emprendimientos analizados, lo hacen por necesidad; encuentran en la posibilidad de crear empresa una solución para la generación de ingresos, suplir las deficiencias de empleo con la fortaleza adicional de lograr su independencia y reconocimiento tanto a nivel personal como de género y social.

A este respecto, merece resaltarse que uno de los aportes de las unidades empresariales analizadas es la generación de empleo autónomo, con un factor adicional, el hecho de trabajar para ellas les facilita disponer tiempo para la atención de sus familias. 
Otra conclusión es que el conocimiento previo y la experiencia de las emprendedoras ha llevado al sostenimiento de sus negocios por un espacio superior a los diez años de existencia, lo cual permite catalogar en términos de la clasificación del GEM, a más de la mitad de las unidades analizadas como empresarias establecidas; aspecto que denota ampliamente que poseen las condiciones de liderazgo e innovación, atribuidas por la mayoría de los autores al emprendimiento.

La influencia familiar también es una de las motivaciones para que la mujer emprenda y se esfuerce por lograr el crecimiento y éxito de su empresa, en la investigación se evidencia que todas las emprendedoras destinan parte de los ingresos del negocio para cubrir los gastos de su familia como educación, salud, recreación y vivienda, aunque la participación de familiares en la empresa es baja esto se relaciona a que por el tamaño de las empresas solo se emplee las mismas propietarias.

La experiencia en el oficio son oportunidades de emprendimiento para las mujeres de la comuna 5, que debido a sus fracasos, vivencias y trabajos anteriores adquirieron conocimientos que hoy les brinda la confianza requerida para materializar la idea de negocio y las herramientas para implementar estrategias exitosas en sus empresas.

Finalmente, como resultado del presente estudio, se pudo observar que el emprendimiento femenino en la comuna cinco (5) de la ciudad de Villavicencio, ha surgido fundamentalmente por necesidad, sin embargo, la influencia del entorno, dado por las condiciones del país, ha impactado las decisiones para dicho emprendimiento y el sostenimiento de las unidades de negocio, convirtiéndolo en oportunidad, cumpliendo una función social muy importante como lo es la generación de empleo, que a su vez ayudó a impulsar a otras mujeres.

No obstante, vale la pena resaltar la existencia de muchas limitaciones para el emprendimiento en Villavicencio, resaltan la falta de apoyo, la presencia estatal y el desconocimiento de las emprendedoras sobre entes que promuevan o fomenten la creación de empresas y que contribuyan para el desarrollo de las ideas de negocio, también cabe señalarse que la aversión a proporcionar información por parte de las mujeres, especialmente de aquellas que han sufrido alguna condición de vulnerabilidad personal o de su seguridad, se constituyó en la mayor limitante para el desarrollo de la investigación, lo que convierte estos aspectos en situación de interés para futuras investigaciones. 


\section{REFERENCIAS}

(1) Asociación Colombiana de las Micro, Pequeñas y Medianas empresas-ACOPI. (2017). Encuesta de desempeño empresarial. Informe de resultados ler trimestre 2017. Recuperado de http://acopi.org.co/wpcontent/uploads/2017/05/INFORME-DE-RESULTADOS-ENCUESTA-1er-TRIMESTRE-DE-2017.pdf

(2) Avolio, B. y Di Laura, G. (2017). Progreso y evolución de la inserción de la mujer en actividades productivas y empresariales en América del Sur. Revista de la CEPAL. 122, 35-62. Recuperado de https://repositorio.cepal.org/bitstream/handle/11362/42031/1/RVE122_Avolio.pdf

(3) Azqueta, A. (2019). Análisis del concepto emprendedor y su incorporación al ámbito educativo. Revista Teoría de la educación, 31(1), 57-80. doi: 10.14201/teri.19756.

(4) Bucardo, M., Saavedra, M. y Camarena, M. (2015). Hacia una compresión de los conceptos de emprendedores y empresarios. Suma de Negocios, 6(13), 98-107 doi: 10.1016/j.sumneg.2015.08.009

(5) Burgos, R., Pombosa, E. y López, L. (2017). Los emprendimientos como elementos de estudio teóricos, académicos e investigativos. Revista contribución a las ciencias sociales. Recuperado de http://www.eumed.net/rev/cccss/2017/02/aporte-emprendimiento.html

(6) Castiblanco, S. (2013). La construcción de la categoría de emprendimiento femenino. Revista Facultad de Ciencias Económicas: Investigación y Reflexión, 21(2), 53-66. Recuperado de http://www.redalyc.org/articulo.oa?id=90930501005

(7) Castro, A., Farfán, D. y Olis, I. (2013), Vida y muerte empresarial. Estudios empíricos de perdurabilidad: el Grupo Nutresa. Revista Lebret, (5), 317-329. doi: 10.15332/rl.v0i5.835

(8) Congreso de la República de Colombia (2004). Ley 905 de 2004 por medio de la cual se modifica la Ley 590 de 2000 sobre promoción del desarrollo de la micro, pequeña y mediana empresa colombiana y se dictan otras disposiciones. Bogotá D.C.: Congreso de la República de Colombia.

(9) Elizundia, M. (2015). Desempeño de nuevos negocios: perspectiva de género. Contaduría y Administración, 60(2), 468-485. doi: 10.1016/S0186-1042(15)30010-3

(10) Global Entrepreneurship Monitor -GEM. (2017). Estudio de la actividad empresarial 2017. Recuperado de https://www.gemconsortium.org/report/gem-colombia-2017-report

(11)León, Y., Aguas, T. y Rojas, A. (2018). Características de las mujeres emprendedoras. (Tesis de pregrado). Universidad Cooperativa de Colombia. Colombia. Recuperado de https://repository.ucc.edu.co/bitstream/20.500.12494/6018/1/2018\%20-

$\% 20$ Aguas\%2C\%20Rojas\%20y\%20Leon\%20\%20-

$\% 20 \% 20$ Caracteristicas\%20de\%20Mujeres\%20Emprendedoras\%20.pdf 
Caracterización y motivaciones para el emprendimiento femenino en MIPYMES de Villavicencio - Colombia

Ruiz Sanchez_ María del Carmen; Peña Guerrero_ Jennifer Vanessa; Prieto Cubillos_ Bryan Leonardo

(12) Marulanda, F., Montoya I. y Vélez J. (2014). Aportes teóricos y empíricos al estudio del emprendedor. Cuadernos de Administración, 30(51), 89-99. Recuperado de http://www.scielo.org.co/pdf/cuadm/v30n51/v30n51a10.pdf

(13) Marulanda, J., Correa, G. y Mejía, L. (2009). Emprendimiento: Visiones desde las teorías del comportamiento humano. Revista Escuela de Administración de Negocios, (66), 153-168. Recuperado de http://www.redalyc.org/articulo.oa?id=20620269008

(14) Navarro, U. (2016). Análisis del emprendimiento femenino atendiendo a la influencia del rol de la mujer en el acceso al mercado laboral y en la educación superior. Opción, 32(10), 394-411. Recuperado de http://www.redalyc.org/articulo.oa?id=31048901021

(15) Obando-Bastidas, J., Herrera-Sarmiento, G. y Rodríguez-Ladino, J. (2016). Los microempresarios y los cuentagotas en Villavicencio. Orinoquia, 20(2), 102-111. doi: 10.22579/20112629.363

(16)OCDE. (2016). OECD Reviews of Labor Market and Social Policies: Colombia 2016. doi: $10.1787 / 9789264244825$-en

(17) Pereira, F. (2003). Reflexión sobre algunas características del espíritu emprendedor colombiano. Revista Economía Gestión y Desarrollo, 1(1), 9-26. Recuperado de: http://revistaeconomia.puj.edu.co/html/articulos/Numero_1/art1.pdf

(18) Pita, S. y Pétergas, S. (2002). Investigación cuantitativa y cualitativa. Cuadernos de atención primaria, 9 , 76-78. Recuperado de http://www.fisterra.com/gestor/upload/guias/cuanti_cuali2.pdf

(19)Real Academia Española. (2018). Diccionario de la lengua española. Recuperado de https://dle.rae.es/?id=Esj9hsT

(20) Secretaría de Planeación Departamento del Meta. (2017). Departamento Administrativo de Planeación. Recuperado en Julio 14 de 2018 de: https://meta.gov.co/secretaria/departamento-administrativo-deplaneacion/16

(21) Simón, V., Revuelto, L. y Medina, A. (2012). La influencia de la formación, la experiencia y la motivación para emprender en la supervivencia de las empresas de nueva creación. Estudios Gerenciales, 28(EE), 237262. doi: 10.18046/j.estger.2012.1487

(22) Ugalde, N. y Balbastre, F. (2013). Investigación cualitativa e investigación cualitativa: buscando las ventajas de las diferentes metodologías de investigación. Ciencias económicas, 31(2). 179-187. Recuperado de https://revistas.ucr.ac.cr/index.php/economicas/article/view/12730/11978

(23) Valencia, G. (2012). Autoempleo y emprendimiento. Una hipótesis de trabajo para explicar una de las estrategias adoptadas por los gobiernos para hacer frente al progreso del mercado. Semestre Económico, 15(32), 103-128. Recuperado de http://www.scielo.org.co/pdf/seec/v15n32/v15n32a5.pdf

(24) Veciana, J. M. (1999). Creación de Empresas como programa de investigación Científica. Revista Europea de Dirección y Economía de la Empresa, 8(3), 11-36. 
(25) Velásquez, J. (2009). El emprendimiento en la teoría económica: Una revisión bibliográfica. doi: 10.13140/RG.2.1.4613.8005

Cómo citar este artículo: Ruiz, M., Peña, J. y Prieto, B. (2020). Caracterización y motivaciones para el emprendimiento femenino en MIPYMES de Villavicencio - Colombia. Tendencias, 21(2), 146-166. https://doi.org/10.22267/rtend.202102.145 